\title{
Stripe domain structures in a thin ferromagnetic film
}

\author{
A. B. Kashuba \\ Landau Institute for Theoretical Physics, Kosygin str. 2, Moscow 117940, Russia \\ V. L. Pokrovsky \\ Landau Institute for Theoretical Physics, Kosygin str. 2, Moscow 117940, Russia \\ and Department of Physics, Texas A $1 M$ University, College Station, Texas 77843-4242
}

(Received 5 March 1993; revised manuscript received 24 May 1993)

\begin{abstract}
We present a theory of the stripe domain structure in a thin ferromagnetic film with single-ion easy-axis magnetic anisotropy and long-range dipole interactions, for a wide range of temperatures and applied magnetic field. The domains exist at temperatures below the reorientational phase transition from out-of-plane to in-plane magnetization. The system of stripes can be described as a liquid crystal with a preferred domain-wall orientation. The positional order is destroyed by both thermodynamical meandering of domain walls and by the proliferation of dislocations. Spatial anisotropy generated by the fourth-order exchange energy stabilizes the stripe domain structure and pins its orientation. For any temperature below the reorientational phase transition there exists a critical perpendicular-to-plane magnetic field, which separates multidomain and monodomain states of the film. The theory explains recent experimental observations.
\end{abstract}

\section{INTRODUCTION}

Recently the physical properties of ultrathin (a few monolayers) ferromagnetic films have been intensely studied both experimemtally and theoretically. In part this is due to the prospect of using such films as highdensity storage devices. Fe and Co films on $\mathrm{Cu}, \mathrm{Ag}$, and Au substrates have a Curie temperature exceeding room temperature and sometimes exceeding the temperature of film evaporation.

Ultrathin films display a remarkable size effect: The direction of magnetization depends crucially on the thickness of the film. In thicker films (ten or more monolayers) the magnetization, if it exists, is parallel to the plane. In extremely thin films (one to five monolayers) the magnetization is always perpendicular to the film. In films of intermediate thickness the magnetization is found to be perpendicular to the plane at low temperatures and parallel at high temperatures. ${ }^{1-3}$ More accurate measurements ${ }^{4}$ have shown the existence of a reorientation phase transition (RPT) in the range of temperatures $230-270 \mathrm{~K}$.

The very existence of a perpendicular magnetization is nontrivial, since the demagnetizing factor of a film favors a parallel magnetization. The cubic symmetry of the fcc iron lattice is broken near the film surface, resulting in uniaxial anisotropy. This effect is stronger the thinner the film. $\mathrm{Néel}^{5}$ argued that the surface-induced anisotropy is of the easy-axis type. His intuitive conclusion has been supported by numerical calculations of Gay and Richter 6 on the ground state of an iron film on a copper substrate. They have concluded that, in the ground state, the magnetization should be directed perpendicular to the film.

Pescia and Pokrovsky ${ }^{7}$ and Teitelman ${ }^{8}$ have suggested that the RPT is the result of a competition of the surface magnetic anisotropy and the dipolar forces, both renormalized by strong thermal fluctuations (see also the discussion in Levanyuk and Garcia ${ }^{9}$ and Pescia and Pokrovsky ${ }^{10}$ ). The effective anisotropy $\lambda_{\text {eff }}$ includes the bare anisotropy $\lambda$ and a contribution from a short-range part of the dipolar interaction, $\Omega_{s}$, which is of opposite sign. The bare anisotropy energy does not depend on the number of layers, whereas the dipolar energy is proportional to the number of layers, $N$, so that the effective anisotropy can be written as

$$
\lambda_{\mathrm{eff}}=\lambda-N \Omega / a,
$$

where $\Omega=4 \pi g^{2} \mu_{B}^{2} / a^{4}$ characterizes the strength of the dipolar interaction. Here $\mu_{B}$ is the Bohr magneton, $g$ is the Landé factor, and $a$ is the lattice constant. The calculations of Gay and Richter ${ }^{6}$ give $\lambda a^{2} \approx 22 \mathrm{~K}(a \approx 3 \AA)$. This energy should be compared with the characteristic dipolar energy $\Omega a \approx 2.4 \mathrm{~K}$. For a proper choice of $N \approx \lambda a / \Omega$ the anisotropy $\lambda_{\text {eff }}$ can be made of the order of $\Omega / a$. For $N>\lambda a / \Omega$ the magnetization lies in the plane at zero temperature.

Thermal fluctuations renormalize $\lambda$ and $\Omega$ in different ways, so that $\lambda$ is decreased with temperature faster than $\Omega$, and $\lambda_{\text {eff }}$ goes to zero at some temperature $T_{r}$. According to Refs. 9 and 10 the leading logarithmic corrections due to classical fluctuations are the same for both $\lambda$ and the short-range part of $\Omega$ and thus cannot change the sign of $\lambda_{\text {eff }}$. However, in the next approximation $\lambda$ decreases with temperature slower than $\Omega$. We have performed explicit perturbational calculations for a case of large spin $S$ and approximate renormalization-group calculations for an arbitrary $S$. In both cases we got the RPT temperature of the order of the Curie temperature, provided $\lambda_{\text {eff }}$ and $\Omega$ are of the same order of magnitude. These calculations will be published elsewhere.

As long as a perpendicular magnetization exists, the dipolar interaction favors the formation of a domain structure. Yafet and Gyorgy ${ }^{11}$ have calculated the energy and period of a stripe domain structure (SDS) and 
have found that it is energetically favored compared to the uniform state. We rederive their results in a simpler way and show that the domain size is too large to be experimentally accessible at low temperatures, whereas it takes on more accessible values near the RPT (Sec. II).

In the experiment of Ref. 4, a range of temperatures of about $20 \mathrm{~K}$ was found where no net magnetization occurs. We conjectured ${ }^{12}$ that this effect can be ascribed to a domain structure penetrating a sample near the RPT. Indeed, Allenspach and Bischof ${ }^{13}(\mathrm{AB})$ have reported the observation of domains in $\mathrm{Fe} / \mathrm{Cu}(001)$ 6.5-monolayer films, using polarized electron microscopy. They have found a well-oriented but positionally disordered SDS in an interval of temperatures between 230 and $280 \mathrm{~K}$. The domains were oriented along the (100) direction. Their sizes do not fluctuate much. Many defects, mainly dislocations, are seen on the electron microscopy photograph. Earlier, observations of a highly irregular domain structure in a $\mathrm{Co} / \mathrm{Au}(100)$ film were reported. ${ }^{14}$

In this article we present a mean-field theory of the SDS in the presence of an external magnetic field perpendicular to the film (Secs. II and III). We calculate the temperature and field dependence of the SDS period, both away from (Sec. II) and near the RPT (Sec. III). In Sec. IV we consider the elasticity of the SDS. We demonstrate in Sec. $\mathrm{V}$ that terms of the fourth order in spatial derivatives of the exchange interaction play an important role both in stabilizing the positional order at low enough temperatures and in pinning the domain orientation. In Secs. VI and VII we consider effects of thermal fluctuations. We show that the regular SDS melts over a wide range of temperatures due to domain-wall meandering and proliferated dislocations. However, at reasonable temperatures the thermal fluctuations do not destroy the orientational order of domain walls. Thus, averaged over many domains, the stripe domain system can be described as a liquid crystal with the nematic ordering.

A brief version of this article has been published elsewhere. ${ }^{15}$

\section{MEAN-FIELD THEORY OF THE DOMAIN STRUCTURES}

We start with a continuous version of the Hamiltonian for a two-dimensional (2D) ferromagnet:

$H=\frac{1}{2} \Gamma(T) \int[\boldsymbol{\nabla} \cdot \mathbf{n}(\mathbf{x})]^{2} d^{2} x-\lambda(T) \int n_{z}^{2}(\mathbf{x}) d^{2} x+\frac{1}{4 \pi} \Omega(T) \iint \frac{\mathbf{n}(\mathbf{x}) \cdot \mathbf{n}\left(\mathbf{x}^{\prime}\right)-3[\nu \cdot \mathbf{n}(\mathbf{x})]\left[\nu \cdot \mathbf{n}\left(\mathbf{x}^{\prime}\right)\right]}{\left|\mathbf{x}-\mathbf{x}^{\prime}\right|^{3}} d^{2} x d^{2} x^{\prime}$

where $\Gamma=(1 / 2) r S^{2} J$ is the exchange energy ( $r$ denotes the number of nearest neighbors, $J$ the exchange constant, and $S$ the spin per atom), $\lambda$ is the single-ion anisotropy constant favoring perpendicular orientation, $\Omega$ is the strength of the dipole interaction (see its definition in the Introduction), $\boldsymbol{\nu}$ is a unit vector pointing in the direction of $\mathbf{x}-\mathbf{x}^{\prime}$, and the integrals in (2) run over the $x-y$ plane defined by the monolayer ( $z$ being perpendicular to this plane). $\mathbf{n}(\mathbf{x})$ is a classical vector field of unit length with three components. The use of a continuum approximation and of classical vectors to represent the spins is justified since the characteristic wavelength entering our problem is always much larger than the lattice constant.

The integration of the dipole interaction in (2) occurs over the range $\left|\mathbf{x}-\mathbf{x}^{\prime}\right|>>\sqrt{\Gamma / \lambda}$. Indeed, as was shown first by Khokhlachev, ${ }^{16}$ the renormalization stops at this scale, since the anisotropy energy becomes equal to the exchange energy. In the $2 \mathrm{D}$ ferromagnet strong thermodynamic fluctuations of the magnetic moments develop at finite temperatures. Motivated by experiments on thin ferromagnetic films, which are usually carried out at room temperature, we must account for these fluctuations. This can be done using the renormalizationgroup approach (see, e.g., Pescia and Pokrovsky ${ }^{7}$ and Teitelman $\left.{ }^{8}\right)$. The renormalized couplings are given by

$$
\begin{aligned}
& \Gamma(T)=\Gamma Z, \quad \lambda(T)=Z^{3}\left(\lambda+\frac{3 \Omega}{2} \int_{0}^{\xi} \frac{e^{-\xi}}{Z^{2}} \frac{d Z}{d \xi} d \xi\right) \\
& \Omega(T)=\Omega Z^{2}, \quad Z=1-\frac{T \xi}{2 \pi \Gamma}, \quad \xi=\frac{1}{2} \ln \frac{\Gamma}{\lambda a^{2}} .
\end{aligned}
$$

The dipole interaction favors the formation of domain walls. Indeed, let us consider a single domain wall and calculate the difference of the dipole energies for this state and for the homogenous state with all spins up. Introducing a coordinate system in the plane with the $x$ axis perpendicular and $y$ axis parallel to the domain wall we express this difference as

$$
\Delta E_{d}=-\frac{\Omega}{\pi} \int_{-\infty}^{0} d x \int_{0}^{\infty} d x^{\prime} \int_{-\infty}^{\infty} d y \int_{-\infty}^{\infty} d y^{\prime}\left[\left(x-x^{\prime}\right)^{2}+\left(y-y^{\prime}\right)^{2}\right]^{-3 / 2}
$$

This integral diverges as the logarithm of the lateral size. Thus $\Delta E_{d}$ can be estimated as $\left(-2 L_{y} \Omega / \pi\right) \ln \left(L_{x} / l\right)$, where $L_{x}$ and $L_{y}$ are the linear sizes of the film in $x$ and $y$ directions, respectively, and $l$ is the domain-wall width. This result implies the ground state of the Hamiltonian (2) to be a multiple-domain structure.
The characteristic scale $L_{d}$, for which the dipole energy becomes comparable with the magnetic exchange energy, is in general much larger than the domain-wall width $l$. Thus one can neglect the dipolar forces in the calculation of the domain-wall spin configuration. It is well known that $l$ and the local contribution to the domain-wall en- 
ergy $E_{s}$ are given by

$$
l=\sqrt{\Gamma / 2 \lambda}, \quad E_{s}=2 \sqrt{2 \Gamma \lambda} .
$$

Let us consider a stripe structure with curved domain walls, as shown in Fig. 1. For the moment we remove all spins from thin stripes (width $l$ ) centered at the soliton lines and assume a perpendicular magnetization for all other spins. The difference between the energy of this structure and the energy of the monodomain state is

$E_{d}=-\frac{\Omega}{\pi} \sum_{k, l} \int_{\Delta_{k}^{+}} d^{2} x \int_{\Delta_{l}^{-}} d^{2} x^{\prime}\left[\left(x-x^{\prime}\right)^{2}+\left(y-y^{\prime}\right)^{2}\right]^{-3 / 2}$,

where $\Delta_{k}^{+}$and $\Delta_{l}^{-}$are the domains with spins up and down, respectively. We have taken the $k$ even and the $l$ to be odd integers. Using Gauss's theorem this integral can be transformed into a line integral over the boundaries $\Gamma_{m}^{ \pm}$of the domains (see Fig. 1):

$E_{d}=-\frac{\Omega}{\pi} \sum_{m, n}(-1)^{m-n} \int_{\Gamma_{m}^{+}} d \mathbf{l}_{m} \int_{\Gamma_{n}^{-}} d \mathbf{l}_{n}\left(R_{m n}\right)^{-1}$,

where $R_{m n}$ is a distance between two points on the contours $\Gamma_{m}^{+}$and $\Gamma_{n}^{-} ; d \mathbf{l}_{m}$ and $d \mathbf{l}_{n}$ are the vector differentials along the contours.

The representation (7) allows a simple electrical anal-

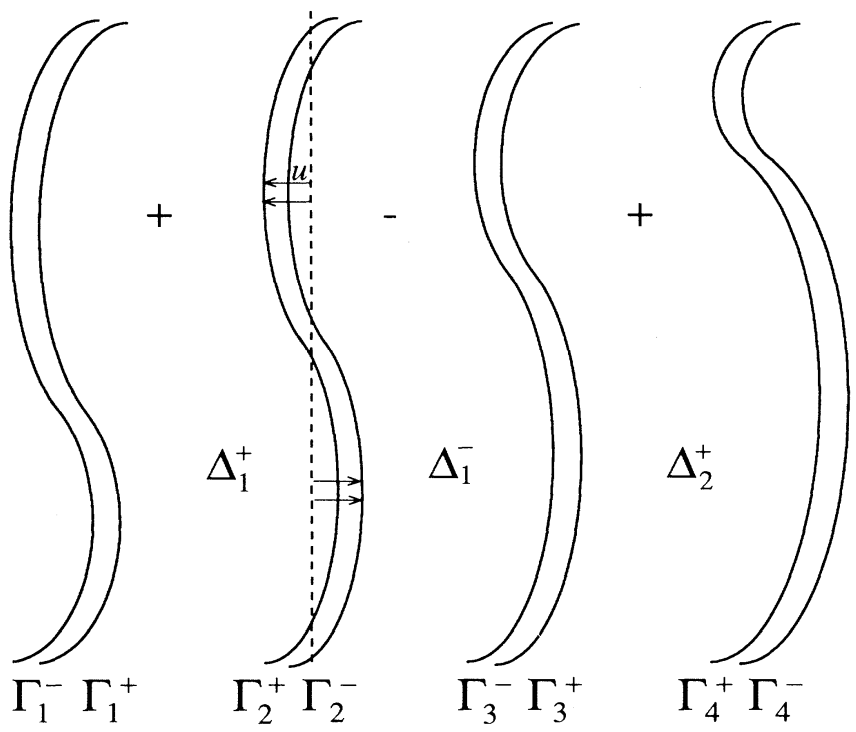

FIG. 1. Schematic representation of a stripe domain structure in a ferromagnetic film. Domains are represented by broad bands. Signs denote the magnetization direction in up $(+)$ and down $(-)$ domains. The $k$ th domain is denoted by $\Delta_{k}^{ \pm}$. Domain walls (of finite width $l$ ) are drawn as narrow bands; in our simplified model they contain no spins. The domain-wall boundaries are denoted by $\Gamma_{k}^{ \pm}$, where \pm corresponds to the sign of the domain they limit. Domain walls are displaced from their ideal position; for the second domain wall, this is represented by the dashed line. The displacement has only one component $u$, which depends on $x$ and $y$, where $x$ is a continuous version of the domain number $k$. ogy. The expression on the right-hand side (RHS) of Eq. (7) is the self-induction of a system of wires, coinciding with the contours $\Gamma$ and carrying electric currents. The remaining part of the dipole interactions, which depends on the particular spin configuration inside the domain walls, is proportional to the overall length of these walls and can be accounted for in our electromagnetic analogy by the magnetic susceptibility of the wire. It will be of no importance to the determination of the magnetic structure and can be incorporated into the energy of the domain wall $E_{s}$. Henceforth we assume that there are no spins at all within the thin stripes of width $l$.

We use Eq. (7) first to calculate the energy per unit area of the regular SDS. Having in mind a magnetic field acting on the film we consider a system of regular alternating stripes with lateral sizes $L+\delta$ and $L-\delta$ for spins up and down, respectively (Fig. 2). Let the $x$ axis be directed perpendicular to the domain walls and the $y$ axis be parallel to them. Then the $x$ coordinates of the boundaries are

$$
x_{2 k}^{ \pm}=2 k L \pm l / 2, \quad x_{2 k+1}^{ \pm}=(2 k+1) L+\delta \pm l / 2 .
$$

The dipolar energy (7) for this special situation reads

$$
\begin{aligned}
H_{d}=-\frac{\Omega}{\pi} N L_{y} \int d y \sum_{j}( & \left(4 L^{2} j^{2}+y^{2}\right)^{-1 / 2} \\
& \left.-\left\{[(2 j+1) L+\delta]^{2}+y^{2}\right\}^{-1 / 2}\right)
\end{aligned}
$$

where $N$ is the total number of domains and $L_{y}$ is the length of any domain wall (the length of the film in the $y$ direction). Here we have performed an integration and summation over the center of the mass coordinate and neglected the difference between $\Gamma_{m}^{+}$and $\Gamma_{m}^{-}$. This approximation is valid when the condition $l \ll L$ is satisfied and $m \neq n$. For the term with $j=0$ the integration must be cut off at $y \approx l$, or equivalently one can integrate $\left(l^{2}+y^{2}\right)^{-1 / 2}$. After the integration and a simple transformation we arrive at a sum:

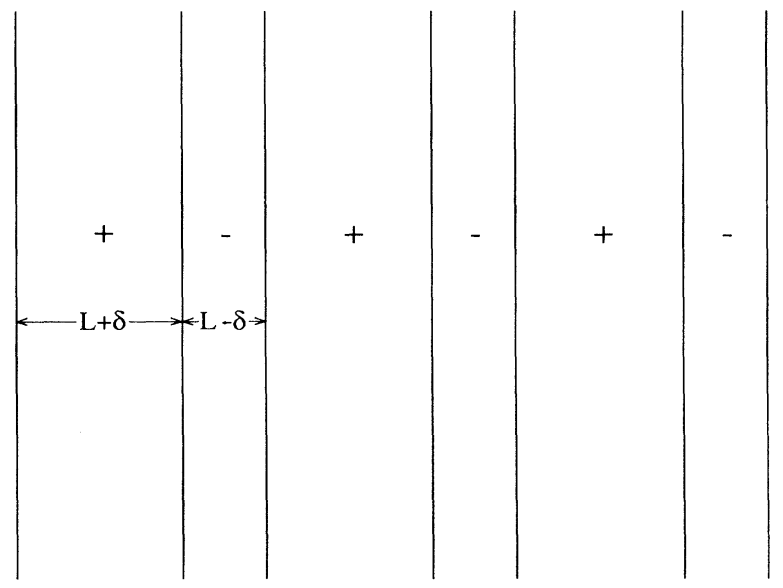

FIG. 2. Schematic representation of the periodic stripe domain structure in the presence of a magnetic field. The domains + and - are not equivalent, having respective widths $L+\delta$ and $L-\delta$. The domain-wall width $l$ is neglected. 


$$
S=\ln \frac{L}{l}+\sum_{j=0}^{\infty} \ln \left(1-\frac{\delta^{2}}{L^{2}(j+1 / 2)^{2}}\right) .
$$

The summation can be performed explicitly:

$$
S=\ln \left(\frac{2 L}{\pi l} \cos \frac{\pi \delta}{2 L}\right) .
$$

Besides the dipolar energy (7) the total energy contains contributions from the short-range energy of the domain walls, equal to $E_{s} \times$ (the total length of domain walls), and from the Zeeman energy of spins in the magnetic field $H$. Collecting all these terms we get the total energy per unit area:

$$
\begin{aligned}
& E=E_{s} n-\frac{\Omega}{\pi} n \ln \left(\frac{2}{\pi n l} \cos \frac{\pi \delta n}{2}\right)-2 h n \delta, \\
& n \equiv \frac{1}{L}, \quad h \equiv g \mu_{B} H,
\end{aligned}
$$

where $n$ is the density of domain walls (the number of walls per unit length). By the derivation Eq. (11) is valid if $L-\delta \gg l$. It can be extrapolated to $L-\delta=l$, where it gives a the reasonable answer $E_{d}=0$.

Minimization of the energy (11) at $h=0$ leads to $\delta=0$ and

$$
n=n^{*}(T)=\frac{\pi}{2 l} \exp \left(-\frac{\pi E_{s}}{\Omega}-1\right) .
$$

Equation (12) shows that the period of the domain structure changes very rapidly with temperature. At low temperature $E_{s}=2 \sqrt{2 \Gamma \lambda}$ is typically much greater than $\Omega$. Thus, the period is astronomically large, and the film remains in the monodomain state. However, near the RPT the effective anisotropy $\lambda$ becomes small, and $L$ acquires a value comparable to or smaller than the size of the film. At this temperature domains enter the film. The strong exponential dependence of size on temperature mimics a sharp phase transition. Thus, the absence of any net magnetization in an interval of temperature about 20-30 $\mathrm{K}$ near the RPT observed in the experiment ${ }^{4}$ can be interpreted as a multidomain structure entering the film in this temperature interval. Since only the total magnetization was measured, one could not observe the magnetization in any individual domain. The existence of the stripe domain structure has been reliably established in the experiments by Allenspach and Bischof. ${ }^{13}$

The energies of various domain structures (striped, triangular, square, etc.) differ only by a numerical factor within the logarithm in Eq. (12). We have performed numerical calculations which show the stripe structure to have the minimal energy.

Equation (12) fails in a small vicinity of the reorientational phase-transition point. Here, the out-of-layer magnetization is far from saturation, and the theory ${ }^{17}$ predicts the period $L$ of the stripe structure to equal the so-called dipole length

$$
L_{d} \equiv \Gamma / \Omega,
$$

for the case of infinitely strong easy-axis anisotropy $\lambda$ (Ising-like model). In Sec. III we find a similar result for an almost isotropic case described by the Hamiltonian (2).

It is useful to note that the largest terms $E_{s} n$ and $-\Omega n \ln n l$ compensate each other at the equilibrium, and comparatively small interactions of the order of $\Omega n$ determine the structure. Therefore higher-order corrections to the exchange interaction must be inspected carefully. This will be done later.

When a nonzero magnetic field $h$ is included the equilibrium conditions read

$$
\delta=\frac{2}{\pi n} \sin ^{-1} \frac{h}{h_{c}}, \quad n=n^{*}(T) \sqrt{1-\left(\frac{h}{h_{c}}\right)^{2}},
$$

where the critical field $h_{c}$ is defined as

$$
h_{c} \equiv 4 \Omega n^{*}(T) \text {. }
$$

At fields higher than critical the film is in the monodomain state. As a consequence of the large characteristic values of $L$ (small $n^{*}$ ), very small magnetic fields can produce a dramatic effect, transforming the multidomain state into the monodomain one. For $L \sim 1 \mu \mathrm{m}$ the characteristic value of $H_{c}$ is estimated as $0.3-1.0 \mathrm{Oe}$.

Experimentally, a remarkable asymmetry of up and down polarization has been observed (AB). It can be explained as the result of a small uncontrolled magnetic field. An alternative explanation would be a hysteresis effect due to domain-wall pinning. This explanation is in contradiction with the clearly reversible magnetization measurements by Pappas, Kaemper, and Hopster. ${ }^{4}$ To settle the issue, measurements in the presence of a controlled magnetic field would be desirable.

Returning to Eq. (14) we find that $L$ and $\delta$ go to infinity at $h \rightarrow h_{c}$, but the difference $L-\delta$ remains finite: $L-\delta \rightarrow 2 /\left(\pi n^{*}\right)$. This behavior agrees qualitatively with the experimental picture (AB).

\section{DOMAIN STRUCTURES IN THE VICINITY OF THE REORIENTATIONAL PHASE TRANSITION (RPT)}

The approximation we have applied in the previous section is obviously invalid for $L \sim l$, i.e., very close to the RPT:

$$
\frac{T_{c}-T}{T_{c}} \leq \frac{\Omega^{2}}{\Gamma \lambda(0)}
$$

In this section we consider the vicinity of the RPT and show that instead of square-shaped domains, a cosinetype modulation occurs which is energy favorable. The period of this modulation tends to a finite limit at the RPT.

Near the RPT the perpendicular magnetization is small, and the spins presumably lie in plane. Nevertheless, there is no average in-plane magnetization. A proper representation of classical spins to account for fluctuations is

$$
\mathbf{S}=\mathbf{n} \sqrt{1-\pi^{2}}+\pi
$$


where $\mathbf{n}$ is a slowly varying unit vector in plane and

$$
\boldsymbol{\pi}=\pi \mathbf{e}+S_{z} \mathbf{e}_{z}
$$

is a small vector perpendicular to $n$. The first term on the RHS of Eq. (18) represents fast fluctuations in plane, and the second represents a perpendicular magnetization with a small, but nonzero average value. Henceforth we neglect the rapid in-plane fluctuations. They are partially accounted for in the renormalized values of $\Gamma$ and the other coupling constants. Furthermore, these fluctuations are suppressed by dipolar forces. Thus, we find the long-wavelength free-energy functional, expanded to the fourth order in $S_{z}$ :

$$
\begin{aligned}
F= & \frac{1}{2} \sum_{q}\left[\Gamma\left(T_{r}\right) q^{2}-\Omega\left(T_{r}\right)|q|-\lambda\right]\left|S_{z q}\right|^{2} \\
& +\frac{\Gamma\left(T_{r}\right)}{2} \int S_{z}^{2}\left(\nabla S_{z}\right)^{2} d^{2} x \\
& +\frac{\Omega\left(T_{r}\right)}{8} \sum_{q} \frac{(\mathbf{n} \cdot \mathbf{q})^{2}}{|q|}\left(S_{z}^{2}\right)_{q}\left(S_{z}^{2}\right)_{-q} .
\end{aligned}
$$

Let us now consider the free energy (19) in the meanfield approximation for $S_{z}$, as a preliminary to performing the averaging over direction of the planar vector $\mathbf{n}$. The quadratic form of $q$ in Eq. (19) has a set of minimal points on a circle of radius $q^{*}=\Omega / 2 \Gamma$. Thus, the Fourier components of the field $S_{z q}$ are restricted to the vicinity of this circle. We compare the free energy of the field $S_{z}$ with the distribution over the circle represented by $2 N$ equidistant $\delta$-function-like peaks. The first configuration $N=1$ corresponds to the stripe domain structure. It has two components in wave-vector space. The second one $(N=2)$ has $C_{4}$ symmetry, and the third one has six components and $C_{6}$ symmetry, etc. The third term of Eq. (19) favors the direction of the domain walls in the stripe structure to be perpendicular to the vector $\mathbf{n}$, whereas the energies of the other structures are invariant with respect to any rotation of the in-plane magnetization n. Below the RPT the third term must be averaged over the direction of the in-plane unit vector $\mathbf{n}$. After minimization of the free energy with respect to the amplitude of the Fourier components, we find

$$
\begin{aligned}
& F=-\frac{\left[\Gamma\left(q-q^{*}\right)^{2}+\delta \lambda\right]^{2}}{4 \Gamma q^{2} S_{N}}, \\
& S_{1}=1, \quad S_{N}=2[N+\operatorname{cotan}(\pi / 4 N) 2 N] \quad(N \neq 1],
\end{aligned}
$$

where $\delta \lambda \sim T_{r}-T$ is a remanent single-ion anisotropy. The stripe structure has the lowest energy among the $C_{N}$ configurations. At nonzero $\delta \lambda$ the energy (20) has to be minimized with respect to the structure wave number $q$. We find

$$
q=q^{*}-\delta \lambda / \Gamma q^{*}, \quad q^{*} \equiv \Omega / 2 \Gamma=1 / 2 L_{d} .
$$

Thus, the period of the stripe structure equals approximately ten dipole lengths $4 \pi \Gamma / \Omega$ at the former transition point $\lambda=0$ and grows rapidly in the vicinity of the reorientational phase transition on the temperature scale $\delta T \sim \frac{\Omega^{2} a^{2}}{\Gamma}\left(\frac{d \lambda}{d T}\right)^{-1}$. Simultaneously the order parameter $\left\langle S_{z}\right\rangle$ increases rapidly, and a cosinelike modulation turns into the SDS. Thus, the cosinelike modulation exists in a rather narrow temperature interval near the RPT. Nevertheless, it has a great significance, limiting the period of the SDS to the value of $L_{d}$. The transition at $T_{r}$ is shifted downward by $\lambda=-\Omega^{2} / 2 \Gamma$.

\section{ELASTIC ENERGY OF THE STRIPE DOMAIN STRUCTURE}

We next discuss thermal fluctuations of the SDS. Two types of fluctuations dominate: the meandering of domain walls and thermally excited dislocations. In both cases the elastic properties of the SDS are substantial. Here we derive the elastic coefficients for the SDS.

Let the domain walls deviate slowly from their ideal positions, as is shown in Fig. 1. We introduce a frame of reference with the $x$ axis perpendicular to and $y$ axis parallel to the domains. A domain-wall displacement can be described by a scalar field $u_{n}(y)$, whose geometrical meaning is illustrated in Fig. 1. In the continuum limit, the domain-wall index $n$ transforms into the continuous variable $x=n L$, and the field $u(x, y)$ defines elastic displacements in two dimensions.

The elastic energy $H_{\mathrm{el}}$ depends on spatial derivatives of $u$, but not on $u$ itself. First we neglect the dependence of the domain-wall energy on its orientation. Then the elastic energy takes the form

$H_{\mathrm{el}}=\int\left\{\frac{K}{2}\left[\frac{\partial u}{\partial x}+\frac{1}{2}\left(\frac{\partial u}{\partial y}\right)^{2}\right]^{2}+\frac{\mu}{2}\left(\frac{\partial^{2} u}{\partial y^{2}}\right)^{2}\right\} d^{2} x$

Landau and Liftshitz have argued $^{18}$ that a term proportional to $(\partial u / \partial y)^{2}$ does not appear in the elastic energy, since $\partial u / \partial y$ is equal to the tangent of the local rotation angle. The first term in (22) is the compression energy, and the expression $\partial u / \partial x+(1 / 2)(\partial u / \partial y)^{2}$ is the compression deformation. The second term is the bending or undulation energy. This special form, including anharmonic terms, has been exploited by Grinstein and Pelcovits. ${ }^{19}$

We will express the elastic coefficients $K$ and $\mu$ in terms of the magnetic constants $\Gamma, \lambda$, and $\Omega$. To this end we use the expression (4) for the dipolar energy, which is valid for an irregular SDS. Together with the local energy of domain walls the total energy reads

$$
\begin{aligned}
H= & E_{s} \sum_{n} \int d y \sqrt{1+\left(\frac{\partial u_{n}}{\partial y}\right)^{2}} \\
& -\sum_{m, n}(-1)^{m-n} \iint V\left(R_{m n}\right) \cos \left(\frac{\partial u_{m}}{\partial y}-\frac{\partial u_{n}}{\partial y^{\prime}}\right) \sqrt{1+\left(\frac{\partial u_{m}}{\partial y}\right)^{2}} \sqrt{1+\left(\frac{\partial u_{n}}{\partial y^{\prime}}\right)^{2}} d y d y^{\prime}
\end{aligned}
$$


where

$$
V(R)=\frac{\Omega}{\pi R}
$$

and

$$
\begin{aligned}
R_{m n}^{2} & =\left[(m-n) L+u_{m}(y)-u_{n}\left(y^{\prime}\right)\right]^{2}+\left(y-y^{\prime}\right)^{2} \\
& =r_{m n}^{2}+2 L(m-n)\left[u_{m}(y)-u_{n}\left(y^{\prime}\right)\right] \\
& +\left[u_{m}(y)-u_{n}\left(y^{\prime}\right)\right]^{2}
\end{aligned}
$$

For a regular structure $\left[u_{n}(y)=0\right]$, minimization of the energy (23) with respect to $L$ gives the equilibrium condition

$$
E_{s}-\sum_{j=-\infty}^{\infty}(-1)^{j} \int d y\left[V(r)-\frac{j^{2} L^{2}}{r} V^{\prime}(r)\right]=0
$$

where $r=\sqrt{j^{2} L^{2}+y^{2}}$.

To find the elastic energy we assume the deformations and bending to be small, and represent the differences of displacements and angles by the expansions

$$
\begin{aligned}
& u_{m}(y)-u_{n}\left(y^{\prime}\right)=(m-n) L \frac{\partial u}{\partial x}+\left(y-y^{\prime}\right) \frac{\partial u}{\partial y} \\
&+\frac{1}{2}\left(y-y^{\prime}\right)^{2} \frac{\partial^{2} u}{\partial y^{2}}+\frac{1}{6}\left(y-y^{\prime}\right)^{3} \frac{\partial^{3} u}{\partial y^{3}} \\
& \frac{\partial u_{m}(y)}{\partial y}-\frac{\partial u_{n}\left(y^{\prime}\right)}{\partial y^{\prime}}=\left(y-y^{\prime}\right) \frac{\partial^{2} u}{\partial y^{2}}
\end{aligned}
$$

All the remaining terms are neglected. Note that the total change of length of the domain walls is proportional to

$$
\int d^{2} x\left[\frac{\partial u}{\partial x}+\frac{1}{2}\left(\frac{\partial u}{\partial y}\right)^{2}\right]
$$

The coefficients of $\frac{\partial u}{\partial x}$ and $\left(\frac{\partial u}{\partial y}\right)^{2}$ turn out to be zero as a consequence of the equilibrium condition (26). The coefficient $K$ can be easily found as a second derivative of the regular SDS energy, Eq. (11):

$$
K=-n^{2} \frac{\partial^{2} E}{\partial n^{2}}=\frac{2 \Omega}{\pi L} .
$$

For the coefficient $\mu$ in (22), a straightforward expansion of (23) gives the expression

$$
\begin{aligned}
\mu=\frac{n}{2} \sum_{j}(-1)^{j} \int d y[ & y^{2} V(r)+\frac{1}{12} \frac{y^{6}}{r^{3}} V^{\prime}(r) \\
& \left.+\frac{1}{12}\left(y^{4}-\frac{y^{6}}{r^{2}}\right) V^{\prime \prime}(r)\right] .
\end{aligned}
$$

The substitution $V(r)=\Omega / \pi r$ is not a trivial operation, because all the integrals over $y$ diverge at any $j$. Therefore the summation over $j$ must be performed first. To this end we represent $r^{-1}$ as a Gaussian integral:

$$
r^{-1}=\frac{1}{\sqrt{\pi}} \int_{-\infty}^{\infty} d \xi \exp \left(-\xi^{2} r^{2}\right)
$$

and apply the Poisson transformation to the sum over $j$. Then Eq. (29) can be rewritten as

$$
\mu=\frac{\Omega}{2 \pi^{3 / 2} L} \sum_{k} \int_{-\infty}^{\infty} d y d z d \xi \exp \left[i(2 k+1) \pi z-\xi^{2}\left(y^{2}+L^{2} z^{2}\right)\right]\left(y^{2}-\frac{1}{3} y^{4} \xi^{2}+\frac{1}{3} y^{6} \xi^{4}\right)
$$

All the Gaussian integrations can be performed explicitly, whereas the summation is expressed in terms of the Riemann $\zeta$ function, leading to

$$
\mu=\frac{21 \zeta(3)}{4 \pi^{3}} \Omega L \approx 0.19 \Omega L
$$

\section{ORIENTATIONAL ANISOTROPY ENERGY}

To complete our discussion of the elastic properties of the SDS we consider the anisotropy energy associated with domain-wall orientation. It originates in the assumed tetragonal symmetry of the spin system on a lattice. The principal term in the exchange interaction [the first term on the RHS of Eq. (2)] does not distinguish between tetragonal symmetry and full rotational symmetry in plane. The first nonvanishing term of the exchange interaction that is sensitive to such a difference is

$$
\delta H=\frac{1}{2} \int \lambda_{a b c d} \frac{\partial^{2} S^{i}}{\partial x_{a} \partial x_{b}} \frac{\partial^{2} S^{i}}{\partial x_{c} \partial x_{d}} d^{2} x,
$$

where by definition the constants $\lambda_{a b c d}$ are invariant under any permutation of four indices. For tetragonal symmetry there are two nonvanishing independent coeffi- cients $\lambda_{x x x x}$ and $\lambda_{x x y y}$. For a generalized Heisenberg model with the Hamiltonian

$$
H=-\sum_{\mathbf{r}, \mathbf{r}^{\prime}} J\left(\mathbf{r}^{\prime}\right) \mathbf{S}_{\mathbf{r}} \mathbf{S}_{\mathbf{r}+\mathbf{r}^{\prime}}
$$

the coefficients $\lambda_{a b c d}$ can be readily calculated:

$$
\lambda_{a b c d}=\frac{1}{a^{2}} \sum_{\mathbf{r}} J(\mathbf{r}) x_{a} x_{b} x_{c} x_{d},
$$

where $a$ is the lattice constant. It is instructive to compare this expression with the analogous formula for the effective exchange constant:

$$
\Gamma=\frac{1}{2 a^{2}} \sum_{\mathbf{r}} J(\mathbf{r}) r^{2}
$$

Clearly the contribution of next-nearest and more remote neighbors to $\lambda_{a b c d}$ is much more substantial than that to $\Gamma$.

Consider now a domain wall whose central line makes an angle $\phi$ with the $x$ axis. Only the components $S_{z}=$ $\cos \theta$ and $S_{x}=\sin \theta$ are supposed to be nonzero in the domain wall, and $\theta$ is taken to be a standard function of a variable $\xi=x \cos \phi+y \sin \phi$ (see Fig. 3 ): 


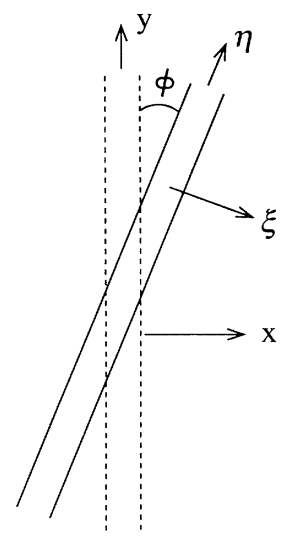

FIG. 3. Schematic representation of a tilted domain wall. Here $\phi$ is the tilt angle, and $x$ is directed perpendicular and $y$ parallel to the preferred domain-wall orientation; $\xi$ and $\eta$ are analogous coordinates associated with the tilted domain wall.

$$
\theta=\cos ^{-1}(\tanh \xi / l)
$$

Substituting (36) into (32) and performing straightforward calculations we get the anisotropic part of the domain-wall energy per unit area:

$$
\delta H_{a}=-\frac{\nu}{16} \cos 4 \phi
$$

where

$$
\nu=8 \frac{3 \lambda_{x x y y}-\lambda_{x x x x}}{L l^{3}} .
$$

Depending on the sign of $\nu$, the domains are oriented preferentially either along the $x$ or $y$ axis or along their bisectors. Considering only small deviations of the orientation from its preferred direction, the anisotropic part of the energy can be rewritten in the form

$$
\delta H_{a}=\frac{|\nu|}{2} \int\left(\frac{\partial u}{\partial y}\right)^{2} d^{2} x
$$

Henceforth we shall write $\nu$ instead $|\nu|$. It is worthwhile to note that neither in-plane anisotropy nor dipolar forces produce an anisotropy of domain-wall orientation unless the discreteness of the lattice is accounted for.

\section{STRIPES AS A LIQUID CRYSTAL}

At temperatures below the RPT, thermal fluctuations influence the stripe domain structure in a crucial way. The striped structure is a particular case of $1 \mathrm{D}$ crystal ordering in two dimensions. Landau and Lifshitz have pointed out (see, e.g., Ref. 18) that thermal fluctuations destroy the long-range order in such systems. In the stripe domain structure there are two main sources of disorder: first, displacements of the domain walls (domainwall meandering); and second, the proliferation of dislocations in the perfect solid, each adding one new semi- infinite stripe. In the presence of quenched impurities, domain walls as well as dislocations can be pinned. Thus, we conclude that the system of stripes can be described either as a liquid or as a glass with a preferred orientation for the domain walls. In other words, it is a kind of a liquid crystal.

Comparing the undulation and orientational anisotropy energies we find that there exists a range of distances

$$
L \ll r \ll L \sqrt{l / a},
$$

where the undulation energy prevails. At larger distances the orientational anisotropy is more important. The value $\sqrt{l / a}$ is not large in real systems (not more than 5-10). Nevertheless, we consider this interval, since this value can vary in different systems, or anisotropy can be suppressed. For example, in a recent experiment by Seul and Wolfe ${ }^{20}$ with a ferrimagnetic garnet film of thickness about $13 \mu \mathrm{m}$, no orientational anisotropy has been found. In such a thick film the magnetization lies in plane, but domains are clearly observable. Orientational anisotropy is expected to be suppressed by thermal fluctuations at high temperature on a large distance scale.

We will shortly show that, if $\nu=0$, the long-range positional order is destroyed by thermal fluctuations at any finite temperature. Moreover, in that case not even algebraic positional order exists. Indeed, in dimension $d=2$, thermal fluctuations lead to a strong divergence of the displacement, i.e., $\left\langle u^{2}\right\rangle \sim R^{3-d}$, where $R$ is the linear size of the film, and so $\left\langle u^{2}\right\rangle \sim R$ for $d=2$. Moreover, dislocations in smectics have a finite energy ${ }^{21,22}$ and therefore their density is finite at any temperature. The dislocation energy can be evaluated as

$$
E_{d}=\frac{K^{3 / 4} \mu^{1 / 4} L^{3 / 2}}{2^{3 / 2} \sqrt{\pi}}
$$

Thus, the SDS is in a liquid state with no positional order. However, even in a liquid state the orientational order persists. One can readily verify that the average square of the angular deviation converges for $d=2$ :

$$
\left\langle\phi^{2}\right\rangle=\left\langle\left(\frac{\partial u}{\partial y}\right)^{2}\right\rangle \sim T \int \frac{p_{y}^{2} d p_{x} d p_{y}}{K p_{x}^{2}+\mu p_{y}^{4}}<\infty .
$$

Only the absence of an infrared divergence must be checked, since there exists a natural ultraviolet cutoff momentum at the inverse lattice constant.

We have thus shown that different pieces of the SDS have a preferred orientation, forming a kind of nematic liquid crystal when averaged over many domains. This type of nematic ordering in positionally disordered smectics was discussed by Toner and Nelson. ${ }^{24}$

Over the range of distances (40) thermal fluctuations yield a strong renormalization of the elastic moduli, which are not constant, but depend on the wave vector in the long-wave limit. Indeed, we will now show that the elastic constant $K$ goes to zero as the wave vector goes to zero. This renormalization was found by Grinstein and Pelcovits ${ }^{19}$ for $d=3$ smectics. The dimension $d=3$ is marginal for a smectic. The dependence of the elastic 
constants on momentum as well as the divergence of the square of the displacement is logarithmic for $d=3$. We use the calculations by Grinstein and Pelcovits to find the elastic constants for $d=2$ by an $\epsilon$ expansion in $(3-\epsilon)$ dimensional space. For the length scale $l$, we find that the invariant charge is

$$
w(l)=\frac{T K^{1 / 2}}{\mu^{3 / 2}} l^{\epsilon} .
$$

It tends to a fixed point at $l \longrightarrow \infty$ :

$$
w_{*}=\frac{64 \pi}{5} \epsilon
$$

This result can be obtained from the Gell-Mann-Low equation for $w$ :

$$
\frac{\partial w}{\partial \xi}=\epsilon w-\frac{5}{64 \pi} w^{2}+O\left(w^{3}\right)
$$

where $\xi=\ln l / a$ and $a$ is the ultraviolet cutoff length (the lattice constant $L$ ). The first term on the RHS of Eq. (45) follows from (43), and the second has been found by Grinstein and Pelcovits. To find the renormalization of the elastic constants we write down the renormalizationgroup equations for the elastic constants $K$ and $\mu$ (Grinstein and Pelcovits have written the equivalent equations for certain combinations of these values):

$$
\begin{gathered}
\frac{d \ln K}{d \xi}=-\frac{w}{16 \pi}, \\
\frac{d \ln \mu}{d \xi}=\frac{w}{32 \pi} .
\end{gathered}
$$

Comparing these equations with Eq. (46) for $w$ we find

$$
\begin{gathered}
K=K_{0}\left(\frac{w}{w_{0}}\right)^{4 / 5}\left(\frac{l}{a}\right)^{-(4 / 5) \epsilon}, \\
\mu=\mu_{0}\left(\frac{w}{w_{0}}\right)^{-2 / 5}\left(\frac{l}{a}\right)^{(2 / 5) \epsilon} .
\end{gathered}
$$

In (49) and (50) we substitute $w=w_{*}=64 \pi / 5 \epsilon$ and $l / a=\Lambda / q$, where $\Lambda=1 / a$ and $q$ is a maximal wave-vector component. More precisely, $q=$ $\max \left[q_{y},\left(\sqrt{K / \mu} q_{x}\right)^{1 / 2}\right]$. Moreover, according to the definition of $w$, Eq. (44), we find

$$
w_{0} \Lambda^{\epsilon}=\left(\frac{K_{0} T^{2}}{\mu_{0}^{3}}\right)^{1 / 2} \text {. }
$$

The final results for $K$ and $\mu$ do not depend explicitly on $\Lambda$ :

$$
\begin{aligned}
& K=K_{0} w_{*}^{4 / 5}\left(\frac{K_{0} T^{2}}{\mu_{0}^{3}}\right)^{-2 / 5} q^{(4 / 5) \epsilon} \\
& \mu=\mu_{0} w_{*}^{-2 / 5}\left(\frac{K_{0} T^{2}}{\mu_{0}^{3}}\right)^{1 / 5} q^{-(2 / 5) \epsilon} .
\end{aligned}
$$

The renormalization changes the relationship between the longitudinal $\left(q_{x}\right)$ and transverse $\left(q_{y}\right)$ components of wave vector. They are balanced at $K q_{x}^{2}=\mu q_{y}^{4}$ or, if we set $\epsilon=1$ in Eqs. (51) and (52),

$$
q_{x} \sim R^{1 / 2} q_{y}^{3 / 2}, \quad R=T / \sqrt{K_{0} \mu_{0}}
$$

where $R$ has the dimensionality of length. Otherwise, one of the components dominates. This result should be compared with the initial relationship $q_{x} \sim \sqrt{\mu_{0} / K_{0}} q_{y}^{2}$. The exact relationship (53) has been recently proved by Golubovic and Wang. ${ }^{23} \mathrm{~A}$ more accurate procedure gives in the first $\epsilon$ approximation $q_{x} \sim q_{y}^{2-(3 / 5) \epsilon}$, slightly differing from (53).

The mean value of the modulus squared of the Fourier transform of the displacement, $\left\langle u_{\mathbf{q}} u_{-\mathbf{q}}\right\rangle$, a quantity of dimension (length) $)^{-4}$, is expressed as a homogeneous function:

$$
\left\langle u_{\mathbf{q}} u_{-\mathbf{q}}\right\rangle^{-1}=\frac{\mu_{0}}{T}\left(\frac{K_{0} T^{2}}{\mu_{0}^{3}}\right)^{1 / 5} q_{y}^{18 / 5} f\left(q_{x} / R^{1 / 2} q_{y}^{3 / 2}\right)
$$

where $f(z)$ is a dimensionless function with asymptotics $f(0)=$ const and $f(z) \sim z^{12 / 5}$ as $z \rightarrow \infty$. The exact result $^{23}$ is $q^{7 / 2}$, instead of $q^{18 / 5}$, in the first $\epsilon$ approximation. Changing (54) to yield the correct exponent of $q_{y}$ leads to

$$
\left\langle u_{\mathbf{q}} u_{-\mathbf{q}}\right\rangle^{-1}=R^{-1 / 2} q_{y}^{7 / 2} f\left(q_{x} / R^{1 / 2} q_{y}^{3 / 2}\right) .
$$

In $x$ space we get a similar result:

$$
\left\langle[u(\mathbf{r})-u(0)]^{2}\right\rangle=R y g\left(R^{1 / 2} x / y^{3 / 2}\right),
$$

where $g(z)$ is again a dimensionless function. The temperature dependence of the correlators was not presented in Ref. 23.

The correlation functions for the order parameter $\exp (i p u)$, where $p=2 \pi / L$, can be calculated in the Gaussian approximation

$$
\left\langle\exp (i p u) \exp \left(i p u^{\prime}\right)\right\rangle=\exp \left(-\frac{p^{2}}{2}\left\langle\left(u-u^{\prime}\right)^{2}\right\rangle\right) .
$$

These predictions of the theory can be checked experimentally. It can be done either by detailed evaluation of electron microphotographs or by optical diffraction measurements. In particular, the theory predicts that the shape of the diffusive diffraction peak is strongly anisotropic, so that the width of the peak in the $x$ and $y$ directions is $p^{3} R^{2}$ and $p^{2} R$, respectively.

For $r \gg L \sqrt{l / a}$ the anisotropy dominates, and the cutoff momentum $q$ in Eqs. (52) and (53) is $\sqrt{\nu / \mu}$. The corresponding scale of length is very large if the substrate has a sixfold symmetry axis [face (111) of $\mathrm{Cu}$ ].

\section{FLUCTUATIONS IN ANISOTROPIC SDS}

The presence of nonzero anisotropy $\nu$ changes the situation drastically. Instead of, for $\nu=0$, dimension $d=3$ being marginal, for $\nu \neq 0$ one has $d=2$ marginal. Long-range order still is destroyed by thermal fluctuations (domain-wall meandering), but algebraic order occurs below the dislocation-mediated melting temperature $T_{m}$ defined as (see, e.g., Lyuksyutov, Naumovets, and Pokrovsky ${ }^{25}$ )

$$
T_{m}=\frac{\sqrt{K \nu}}{2 \pi} L^{2}
$$


The value $T_{m}$ obtained as a solution of this equation is not very reliable since we cannot find the dependence of $L$ on $T$ with the necessary precision. However, Eq. (57) permits us to estimate $L$ at the melting point. Taking $T_{m} \sim \Gamma$ and, by (35), $\lambda_{x x x x} \sim \Gamma a^{2}$ we find $L\left(T=T_{m}\right) \sim 2 \pi L_{d} \sqrt{l / a}$, where $L_{d}$ is given in (13), i.e., between $10 L_{d}$ and $100 L_{d}$ at the melting point. In the experiment by $\mathrm{AB}$ the range of variation of $L$ was about (5-20) $L_{d}$. We cannot tell definitely whether they have reached the melting point. Probably they dealt with the melted phase, i.e., with a liquid crystal of domain walls. Visually we were able to find several dislocations in their photograph. The analysis of dislocation correlations requires more statistics.

In the liquid phase correlations of the order parameter decay exponentially:

$$
G(\mathbf{r}) \sim \exp \left(-r / r_{c}\right), \quad r=\sqrt{x^{2}+(K / \nu) y^{2}},
$$

where the correlation length $r_{c}$ is equal in order of magnitude to the average distance between unbounded dislocations. In the crystal phase, algebraic decay of the correlation takes place:

$$
G(\mathbf{r}) \sim r^{-\frac{\pi T}{2 \sqrt{K \nu} L^{2}}} .
$$

These predictions of the theory can be checked by a careful reexamination of the experimental data.

Finally, note that anisotropy pins the domain wall direction, as has been discussed earlier (see Sec. IV). Here we consider the same effect in a multilayer film. For a quantitative estimate we apply a generalized Heisenberg model (34) with $J(\mathbf{r})=-J_{0} \exp (-\alpha r)$ and Eq. (35) for $\lambda_{\text {abcd }}$. For small enough $\alpha$ the summation in (35) can be replaced by integration with the result

$$
\lambda_{x x x x}=3 \lambda_{x x y y}=-576 \pi J_{0} \alpha^{-7} .
$$

In this approximation the difference $\Delta=\lambda_{x x x x}-3 \lambda_{x x y y}$ is exactly zero. Corrections for the discreteness can be readily made. As a result we get $\Delta>0$. In the opposite limiting case only the nearest-neighbour interaction must be accounted for. Again we get $\Delta>0$. However, numerical estimates show that $\Delta$ changes its sign at some intermediate value of $\alpha$. Taking in account only 4 coordination spheres the value of $\Delta$ can be defined as

$\Delta=J_{0}\left[4 e^{-\alpha r}-32 e^{-\sqrt{2} \alpha r}-72 e^{-\sqrt{3} \alpha r}+4 e^{2 \alpha r}\right]$.

A reasonable estimate of $e^{-\alpha r}$ is about $1 / 30$ (Curie temperature of about $300 \mathrm{~K})$. Then $\Delta$ is negative, and the neglected terms in (61) are about $10 \%$ of $\Delta$. This tendency is strengthened by a tetragonal distortion of the film, which shortens the out-of plane bonds and thus enhances their negative contribution to $\Delta$. Thus, our theory predicts the orientation of the domain walls to be along (100) or (010) directions in the tetragonally distorted fcc film of $\mathrm{Fe} / \mathrm{Cu}(001)$, which indeed has been observed experimentally.

The orientational anisotropy is much weaker by a factor of approximately $10^{4}$ for a hexagonal substrate [face (111) of $\mathrm{Cu}$, since it is associated with higher derivatives of the magnetization.

\section{CONCLUSIONS}

In conclusion we have established that, over a wide range of temperatures below the reorientation phase transition, a domain-wall structure should positionally melt, whereas its orientational order should survive. Thus it exhibits the same properties as a $2 \mathrm{D}$ liquid crystal. The domain-wall anisotropy coming from the exchange energy expanded to fourth order in the spin field derivatives stabilizes the stripe domain structure and forces it to become a solid at some lower temperature.

The characteristic scale of the domain structure is the dipole length $L_{d}$ of Eq. (13), which is usually of the order of magnitude $1 \mu \mathrm{m}$, and increases rapidly with decreasing temperature. Therefore a rather weak magnetic field perpendicular to the film produces a dramatic effect. In particular, it favors a single-domain regime at a critical value $H_{c} \sim 0.1$ Oe.

The most important problem not treated in this work is the influence of quenched defects. We expect a transition to a domain glass state for rapid cooling.

Recent experimental observations (AB) of iron films on the $\mathrm{Cu}(001)$ surface using a polarized electron microscopy method agree in their principal features with the present theory. They display a positionally disordered, but obviously orientationally ordered arrays of domains. The lateral size of the domain structure is indeed in the range of $L_{d}$ near the reorientation phase transition and increases with decreasing temperature.

The problem of domain-wall orientation is more complicated. The domain walls in the experiment are aligned along the (001) direction. In Sec. VII we have presented some arguments in favor of this orientation based on the in-plane next-nearest-neighbor exchange interaction as well as the out-of-plane exchange interactions.

The absence of a prefered orientation in $\mathrm{Co} / \mathrm{Cu}(110)$ film ${ }^{14}$ can be interpreted to indicate an orientationally melted phase. It could mean that in the Co film all the magnetic forces, and particularly the elastic constants of the domain lattice, are weaker than those in the Fe film.

The exposure time in the experiments of $A B$ was not less than $3 \mathrm{~min}$. Hence the observed structure is most probably a glass of domain walls (domain glass). On the other hand, the measurements of magnetization ${ }^{4}$ do not show any notable hysteresis. We conclude that the glassy state of the domain system, if it exists, displays itself in dynamic phenomena on a scale shorter than or of the order of $1 \mathrm{~min}$. Still we do not consider the reversibility of the domain-wall movements to be convincingly proven.

Further research, both experimental and theoretical, is necessary to find the glass transition and regular structures, to find the phase diagram in the $H$ - $T$ plane, to produce controllable measurements in weak magnetic fields, and to find the positional and orientational correlations of the domain walls.

\section{Note added in proof}

After submission of this manuscript, the experimental article by Z. Q. Qiu et al. [Phys. Rev. Lett. 70, 1006 (1993)] has been published. The authors measured the 
magnetization with high precision. They have found a nonzero but small magnetization in the interval where Pappas, Kaemper, and Hopster ${ }^{4}$ did not observe any magnetization. It supports our idea about an uncontrolled magnetic field affecting the system. Another important observation is the curve of the magnetization vs temperature, which is explained by our Eq. (1).

Professor D. Vanderbilt kindly informed us that the result equivalent to our Eq. (11) has been previously obtained by V. Marchenko (Zh. Eksp. Teor. Fiz. 80, 754 (1981) [Sov. Phys. JETP 53, 381 (1981)]) and by D.
Vanderbilt et al. [J. Vac. Sci. Technol. B 7, 1013 (1989); Surf. Sci. Lett. 268, 300 (1992)] for a system of steps on a crystal surface interacting via elastic or electric dipolar forces through the bulk.

\section{ACKNOWLEDGMENTS}

Our thanks are due to R. Allenspach and A. Bischof for sending us their experimental results prior to publication and for discussions. We thank W.M. Saslow for a critical reading of the manuscript and for useful remarks.
${ }^{1}$ J.J. Krebs, B.T. Jonker, and G.A. Prinz, J. Appl. Phys. 63, 3467 (1988).

${ }^{2}$ M. Stampanoni, Phys. Rev. Lett. 59, 2483 (1987).

${ }^{3}$ D. Pescia, Phys. Rev. Lett. 58, 2126 (1987).

${ }^{4}$ D.P. Pappas, K.P. Kaemper, and H. Hopster, Phys. Rev. Lett. 64, 3179 (1990).

${ }^{5}$ L. Néel, J. Phys. Radiat. 15, 376 (1954).

${ }^{6}$ J.G. Gay and R. Richter, Phys. Rev. Lett. 56, 2728 (1986).

${ }^{7}$ D. Pescia and V.L. Pokrovsky, Phys. Rev. Lett. 65, 2599 (1990).

${ }^{8}$ M.G. Teitelman, Zh. Eksp. Teor. Fiz. 98, 1003 (1990).

${ }^{9}$ A.P. Levanyuk and N. Garcia, Phys. Rev. Lett. 70, 1184 (1993).

${ }^{10}$ D. Pescia and V.L. Pokrovsky, Phys. Rev. Lett. 70, 1185 (1993).

${ }^{11}$ Y. Yafet and E.M. Gyorgy, Phys. Rev. B 38, 9145 (1988).

${ }^{12}$ V.L. Pokrovsky (unpublished).

${ }^{13}$ R. Allenspach and A. Bischof, Phys. Rev. Lett. 69, 3385 (1992).

${ }^{14}$ R. Allenspach, M. Stampanoni, and A. Bischof, Phys. Rev.
Lett. 65, 3344 (1990).

${ }^{15}$ A.B. Kashuba and V.L. Pokrovsky, Phys. Rev. Lett. 70, 3155 (1993).

${ }^{16}$ S.B. Khokhlachev, Zh. Eksp. Teor. Fiz. 71, 812 (1976) [Sov. Phys. JETP 44, 427 (1976)].

${ }^{17}$ R. Czech and J. Villain, J. Phys. Condens. Matter 1, 619 (1989).

${ }^{18}$ L.D. Landau and E.M. Lifshitz, Statistical Physics (Pergamon, London, 1977), see Chap. 13.

${ }^{19}$ G. Grinstein and R.A. Pelcovits, Phys. Rev. A 26, 915 (1982).

${ }^{20}$ M. Seul and R. Wolfe, Phys. Rev. Lett. 68, 2460 (1992).

${ }^{21}$ P.G. De Gennes, Solid State Commun. 10, 753 (1972).

${ }^{22}$ P. Pershan, J. Appl. Phys. 45, 1590 (1973).

${ }^{23}$ L. Golubovic and Z.G. Wang, Phys. Rev. Lett. 69, 2535 (1992).

${ }^{24}$ J. Toner and D.R. Nelson, Phys. Rev. B 23, 316 (1981).

${ }^{25}$ I. Lyuksyutov, A.G. Naumovets, and V. Pokrovsky, TwoDimensional Crystals (Academic, San Diego, CA, 1992). 